\title{
COMPOSIÇÃO gUÍMICA DOS FRUTOS DE Campomanesia adamantium (Cambessédes) O.BERG ${ }^{1}$
}

\author{
Maria Isabel VALLILO ${ }^{2, *}$, Leda Conceição Antonia LAMARDO ${ }^{3}$, Maria Lima GABERLOTTI ${ }^{3}$, \\ Elisabeth de OLIVEIRA ${ }^{4}$, Paulo Roberto Hrihorowitsch MORENO ${ }^{4}$
}

\begin{abstract}
RESUMO
Frutos de C. adamantium (Myrtaceae), coletados em diferentes estádios de amadurecimento na Floresta Estadual de Assis - São Paulo -, foram avaliados quanto à composição nutricional, perfil de óleo volátil e presença de elementos inorgânicos. O fruto apresentou alto teor de água (75,9\%), baixo $\mathrm{pH}(4,3)$ e elevada acidez (1,2 g em ácido cítrico). Além disso, destacou-se como excelente fonte de vitamina C (234 mg $100 \mathrm{~g}^{-1}$ ). No óleo volátil, foi possível identificar 40 componentes que corresponderam a 93,3\% dos compostos presentes. Como componentes majoritários do óleo, destacaram-se o $\alpha$-pineno (10,6\%), limoneno (10,1\%) e o $\beta$-(z)-ocimeno (9,2\%). Entre os minerais (16), os principais constituintes foram o K (1304 mg kg-1), Ca, P e Mg na concentração compreendida entre 165 e $175 \mathrm{mg} \mathrm{kg}^{-1}$ e, como micro elementos, o Fe $\left(11,3 \mathrm{mg} \mathrm{kg}^{-1}\right)$ e o $\mathrm{Al}\left(15,9 \mathrm{mg} \mathrm{kg}^{-1}\right)$. O valor energético do fruto $\left(66,3 \mathrm{kcal}^{100} \mathrm{~g}^{-1}\right)$ deveu-se quase que exclusivamente aos carboidratos totais $(11,6 \%)$.

Palavras-chave: Myrtaceae, gabiroba, composição centesimal, valor calórico, óleo essencial.
\end{abstract}

\section{SUMMARY}

CHEMICAL COMPOSITION OF Campomanesia adamantium (Cambessédes) O.BERG' FRUITS. Fruits of C. adamantium (Myrtaceae) collected in different stages of maturation at Assis State Forest, São Paulo, were evaluated for nutritional composition, volatile oil profile and presence of inorganic elements. The fruit showed a high level of moisture (75.9\%), low pH (4.3) and high acidity (1.2 g in citric acid). It is a significant source of vitamin C (234 mg $\left.100 \mathrm{~g}^{-1}\right)$. In the volatile oil, 40 compounds were identified corresponding to $93.3 \%$ of the oil contents. The major components were $\alpha$-pinene (10.6\%), limonene (10.1\%) and the $\beta$ - $(z)$-ocimene (9.2\%). Among the inorganic elements the nutrients exceed: $\mathrm{K}\left(1304 \mathrm{mg} \mathrm{kg}^{-1}\right), \mathrm{Ca}, \mathrm{P}$, and $\mathrm{Mg}$ from 165 to $175 \mathrm{mg} \mathrm{kg}^{-1}$ concentration and the microelements Fe (11.3 $\left.\mathrm{mg} \mathrm{kg}^{-1}\right)$ and $\mathrm{Al}\left(15.9 \mathrm{mg} \mathrm{kg}^{-1}\right)$. The caloric value $\left(66.3 \mathrm{kcal} 100 \mathrm{~g}^{-1}\right)$ was due almost exclusively to the total carbohydrates (11.6\%).

Keywords: Myrtaceae, gabiroba, centensimal composition, caloric value, essential oil.

\section{1 - INTRODUÇÃO}

Os frutos de C. adamantium, popularmente conhecidos como "gabiroba" ou "gabiroba-do-mato", apresentam formato redondo, de coloração que varia do verde escuro ao verde claro e amarelo, e exalam aroma cítrico, agradável ao olfato. Essa espécie pertence à família Myrtaceae, composta por mais de 100 gêneros e 3.600 espécies. A planta se desenvolve de forma arbustiva, atingindo até $2 \mathrm{~m}$ de altura, muito ramificada e com ramos delgados. Suas folhas são simples, opostas, ovais ou elípticas, membranáceas ou cartáceas, com base aguda à obtusa, apresentando ápice agudo com cerca de $4 \mathrm{~cm}$ de comprimento e $2 \mathrm{~cm}$ de largura [14].

A planta floresce nos meses de setembro a novembro, com flores brancas, solitárias, axilares ou terminais. Os

\footnotetext{
${ }^{1}$ Recebido para publicação em 25/11/2005. Aceito para publicação em 20/10/2006 (001644)

Instituto Florestal, Divisão de Dasonomia, Seção de Madeira e Produtos Florestais, Laboratório de Fitoquímica, Rua do Horto, 931, CEP 02377-000, Jd. Tremembé, São Paulo (SP), Brasil

E-mail:maria-vallilo@ig.com.br

${ }^{3}$ Instituto Adolfo Lutz, C. P. 355, CEP 01246-902, São Paulo, Brasil

E-mail:llmardo@ial.sp.gov.br

${ }^{4}$ Instituto de Química, Universidade de São Paulo, C. P. 26077,

CEP 05513-970, São Paulo (SP), Brasil

E-mail:edolivei@iq.usp.br,prmoreno@iq.usp.br

* A quem a correspondência deve ser enviada
}

frutos amadurecem de novembro a dezembro, sendo comestíveis e consumidos por várias espécies de pássaros $\mathrm{e}$ mamíferos [8, 25].

Citações da literatura indicam a ocorrência da espécie em campos de cerrado, no Centro - Oeste e no Sudeste do Brasil e, em alguns casos, chegando a ultrapassar os limites do País para alcançar as terras do Uruguai, Argentina e Paraguai [2, 14, 25].

De acordo com CARRARA [7], as espécies de Campomanesia têm importância econômica bastante diversificada, sendo que alguns dos seus frutos, além de serem consumidos "in natura", também são utilizados na forma de doces, sorvetes, refrescos e, muitas vezes, como flavorizantes em destilados alcoólicos.

Um estudo realizado com os frutos de outra espécie desse mesmo gênero, a $C$. phaea (cambuci), revelou altos teores de umidade $(88,33 \%)$ e acidez $(\mathrm{pH}=2,85)$, baixos teores de proteína $(0,62 \%)$; carboidratos totais $(4,68 \%)$; fibras alimentares (3,80\%); e ácido ascórbico (33,37 mg $\left.100 \mathrm{~g}^{-1}\right)$. Em relação aos elementos inorgânicos, o estudo destacou o sódio (171,50 $\left.\mathrm{mg} \mathrm{kg}^{-1}\right)$; potássio $\left(622,65 \mathrm{mg} \mathrm{kg}^{-1}\right)$; fósforo (123,69 $\left.\mathrm{mg} \mathrm{kg}^{-1}\right)$; magnésio (42,08 $\mathrm{mg} \mathrm{kg}^{-1}$ ); e cálcio $\left(61,26 \mathrm{mg} \mathrm{kg}^{-1}\right)$ [30].

Dessa forma, a falta de conhecimento da composição química dos frutos dessa espécie botânica motivou-nos a avaliar sua composição centesimal, valor nutricional e 
composição química do óleo essencial, visando seu aproveitamento na indústria de alimentos e bebidas.

Os dados obtidos foram comparados com valores encontrados na literatura para frutos comestíveis.

\section{2 - MATERIAL E MÉTODOS}

Frutos inteiros de C. adamantium (aproximadamente $2 \mathrm{~kg}$ ), em diversas fases de amadurecimento, foram coletados de várias plantas, em novembro de 2004, na Floresta Estadual de Assis, município de Assis, na região oeste do Estado de São Paulo, situada entre as coordenadas geográficas de $23^{\circ} 35^{\prime}$ de latitude Sul e $50^{\circ} 22^{\prime}$ de longitude Oeste de Greenwhich. A vegetação original é típica dos cerrados, predominando a forma "cerradão" [17, 18].

O material vegetal estudado foi identificado no Herbário D. Bento Pickel, do Instituto Florestal de São Paulo, recebendo a exsicata o número SPSF 33.729.

O solo da região é classificado em dois tipos: latossolo vermelho-escuro álico, a moderado, textura média LE1, e podzólio vermelho amarelo eutrófico Tb, abrupto, a moderado, textura areno média-PV2. São solos ácidos e de baixa fertilidade, com elevados teores de alumínio [13, 17].

Para as análises químicas, os frutos foram misturados aleatoriamente formando uma amostra composta. Em seguida, foram acondicionados em sacos de polietileno e conservados em freezer, para posteriores análises nos laboratórios do Instituto Florestal, Adolfo Lutz e Instituto de Química da Universidade de São Paulo. Uma alíquota dessa amostra foi triturada e homogeneizada integralmente, em multiprocessador, para posteriores análises químicas Os procedimentos foram feitos segundo as "Normas Analíticas do Instituto Adolfo Lutz" [20], sendo as fibras alimentares totais dosadas pelo método enzimático-gravimétrico da "Association of Official Analytical Chemists - AOAC", modificado por LEE, PROSKY \& DEVRIES [24]; e o ácido ascórbico (vitamina C), dosado pelo método descrito por CONTRERAS-GUZMÁN, STRONGIII \& GUERNELLI [10].

O valor calórico foi calculado utilizando-se os seguintes fatores clássicos de conversão de Atwater: 9 kcal por g de lipídios, $4 \mathrm{kcal}$ por g de proteínas e $4 \mathrm{kcal}$ por g de carboidratos [12].

Para extração dos componentes voláteis, aproximadamente $1.239 \mathrm{~g}$ de amostra já processada foi submetida à técnica tradicional de arraste com vapor d'água, por cerca de $5 \mathrm{~h}$, pelo aparelho de Clevenger modificado por WASICKY [31]. Para a análise, o óleo obtido por hidrodestilação foi diluído em éter etílico na razão de 2:100 (v/v). Os componentes foram quantificados por cromatografia gasosa com detector de ionização de chamas (CG/DIC) e identificados por cromatografia gasosa acoplada à espectrometria de massas (CG/EM). A metodologia empregada para ambas as análises foi descrita por Adams [1]. A análise foi feita em um cromatógrafo a gás Shimadzu GC 17A, equipado com coluna capilar de sílica fundida DB5 (25 m de comprimento e $0,25 \mathrm{~mm}$ de diâmetro interno, preenchida com polidime- tildifenilsiloxano, contendo 5\% de grupamentos fenila com um filme de 0,25 $\mu \mathrm{m}$ de espessura) para a separação dos constituintes. O injetor (com divisão de fluxo - split/splitless) já foi programado para $220^{\circ} \mathrm{C}$ (razão de divisão $1: 20$ ) e a programação de temperatura foi de 40 a $240{ }^{\circ} \mathrm{C}$ a $3{ }^{\circ} \mathrm{C} / \mathrm{min}$ (tempo total de análise $80 \mathrm{~min}$ ), utilizando hélio como gás de arraste a uma pressão de $80 \mathrm{Kpa}$ e velocidade linear de $1 \mathrm{~mL}$ por minuto. Nitrogênio, ar sintético e hidrogênio foram utilizados como gases auxiliares, na razão de 1:1:10, respectivamente. A composição percentual foi obtida por integração eletrônica utilizando software CR10 (Shimadzu) e detector por ionização de chama (DIC, $290{ }^{\circ} \mathrm{C}$ ).

A composição do óleo foi analisada por CG/EM utilizando aparelho Agilent 6890 com quadrupolo cilíndrico, equipado com coluna HP-5 (30 m, 0,25 mm de diâmetro interno e filme de 0,25 $\mu \mathrm{m}$ de espessura), operando com energia de ionização de $70 \mathrm{eV}$ e uma temperatura de interface de $250{ }^{\circ} \mathrm{C}$. A temperatura foi programada de 40 a $240{ }^{\circ} \mathrm{C}$ a $3{ }^{\circ} \mathrm{C} / \mathrm{min}$ (tempo total de análise 80 minutos), utilizando hélio como gás de arraste a um fluxo de $1 \mathrm{~mL} / \mathrm{min}$.

A identificação dos componentes do óleo foi baseada na comparação entre o índice de retenção e o espectro de massas, com amostras autênticas e os dados retirados da literatura [1], ou, ainda, por comparação com espectros de massas registrados em banco de dados como NIST $62 \mathrm{e}$ NIST 12 (National Institute of Standars and Technology), no qual são registrados mais de 65.000 compostos.

A determinação dos elementos inorgânicos ( $\mathrm{Na}, \mathrm{K}, \mathrm{P}, \mathrm{Ca}$, $\mathrm{Mg}, \mathrm{Ba}, \mathrm{S}, \mathrm{Mn}, \mathrm{Fe}, \mathrm{Ni}, \mathrm{Cu}, \mathrm{Se}, \mathrm{Zn}, \mathrm{Pb}$ e $\mathrm{Al}$ ) foi realizada no laboratório de espectrometria do Instituto de Química da Universidade de São Paulo, pela digestão ácida da amostra $\left(\mathrm{HNO}_{3}\right.$ a $\left.30 \%\right)$, em sistema focalizado Spex 350, assistida por radiação de microondas, com três repetições analíticas e determinação por ICP-OES, conforme descrito por VALLILO et al. [29].

\section{3 - RESULTADOS E DISCUSSÃO}

A composição nutricional dos frutos inteiros de $C$. adamantium (gabiroba) está descrita na Tabela 1. Merecem destaque os teores de umidade $(75,9 \%)$, carboidratos ( $11,6 \%)$, proteínas $(1,6 \%)$, fibra alimentar $(9,0 \%)$ e, principalmente, ácido ascórbico (234 mg $100 \mathrm{~g}^{-1}$ ).

Sendo essa espécie encontrada em regiões típicas de cerrado, cujos solos são ácidos e pobres em elementos inorgânicos, sua composição nutricional se mostrou mais rica quando comparada com a espécie do mesmo gênero (C. phaea, cambuci) encontrada em floresta úmida da Mata Atlântica. Esse fato pode ser constatado nos valores obtidos para os carboidratos e proteína, superiores aos do cambuci, e que lhe conferem um maior valor energético (66,3 kcal $\left.100 \mathrm{~g}^{-1}\right)$.

Destaca-se, também, o alto teor de ácido ascórbico encontrado, muito superior aos teores do kiwi ((98,0 mg $\left.100 \mathrm{~g}^{-1}\right)$, pitanga (14,0 mg $\left.100 \mathrm{~g}^{-1}\right)$, goiaba vermelha $(40,0$ $80,1 \mathrm{mg} 100 \mathrm{~g}^{-1}$ ), que são espécies da mesma família botânica, com exceção do kiwi, e relatadas na literatura [5, 15, 
$22,28]$, tendo, aproximadamente, teor sete vezes superior ao do cambuci (Tabela 1$)$.

Segundo LARCHE [23], o pH do solo tem efeito direto sobre a viabilidade dessas plantas, além de seu efeito sobre a provisão de nutrientes. Este autor observou que o baixo teor de fósforo no solo onde ocorriam espécies de Campomanesia, pode ter influenciado a floração. Como este elemento está intimamente ligado à essa fase fisiológica da planta, pode ter contribuído, por exemplo, para a ausência deste fato em C. xantocarpa, que foi acompanhada pelo autor por cerca de um ano e não floresceu. A ausência ou presença de floração e frutificação também sofre influência direta de fatores como fotoperíodo, pluviosidade e temperatura.

A concentração de ácido ascórbico mostrou que os frutos da gabiroba são importante fonte natural de vitamina C para a avifauna e para os habitantes da região. Para o consumo humano, o Ministério da Saúde recomenda a ingestão diária de $60 \mathrm{mg}$ de vitamina C para adultos [3]. Nesse sentido, verificou-se que cada fruto de gabiroba da região estudada, pesando em média 2,3 g, forneceu 5,4 mg de vitamina $\mathrm{C}$. Dessa forma, pode-se inferir que a ingestão aproximada de 12 frutos supre o teor recomendado pela legislação brasileira para a dieta alimentar diária de adultos.

Os frutos se apresentaram com sabor ácido $(\mathrm{pH}=4,3)$, aroma cítrico, persistente e agradável ao olfato. A presença de traços de óleo essencial, de cor amarela, correspondendo a $0,06 \%(\mathrm{v} / \mathrm{p})$, contribuiu para o aroma característico dos frutos.

Pela análise cromatográfica e dos dados de espectrometria de massas, pôde-se identificar e quantificar 40 componentes no óleo volátil, que corresponderam a 93,3\% dos compostos presentes (Tabela 2). Os componentes majoritários deste óleo foram os hidrocarbonetos monoterpênicos $\alpha$-pineno ( $10,6 \%)$, limoneno (10,1\%) e $\beta$-(z)-ocimeno $(9,2 \%)$. A presença desses compostos no óleo volátil pode ser um dos fatores responsáveis pelo aroma cítrico observado nos frutos, principalmente o limoneno, o qual é o componente majoritário do óleo volátil em várias espécies de Citrus.

Entre os sesquiterpenos, destacam-se o $\beta$-cariofileno $(7,04 \%)$, o ledeno $(5,16 \%)$ e o globulol $(3,18 \%)$. Contudo, a análise completa do aroma deste fruto não foi contemplada neste trabalho, pois este pode conter ainda vários compostos voláteis aromáticos que não são extraídos pela metodologia empregada. Sabe-se pela literatura que o aroma típico das frutas tropicais é resultado da combinação de inúmeras substâncias voláteis representantes de diversas classes de compostos orgânicos, com diferentes propriedades físicoquímicas e que, em sua maioria, são substâncias termolábeis, sujeitas, portanto, a rearranjos, ciclizações e oxidações quando submetidas ao aumento de temperatura [16].

Dados da literatura enfatizam os resultados positivos de algumas dessas substâncias, no tratamento de tumores malignos, quando administradas isoladamente na dieta alimentar de roedores. É o caso do limoneno na sua forma enantiômera (D-limoneno), na terapia de câncer de mama, pâncreas e próstata $[11,21]$ e do $\beta$-cariofileno que apresenta atividade espasmolítica [6], anestésica local [19] e antiinflamatória [27].

Em relação aos elementos inorgânicos (Tabela 3), os frutos de gabiroba concentram em maior quantidade os macroelementos $\mathrm{K}$ (1304 $\mathrm{mg} \mathrm{kg}^{-1}$ ), Ca, P e Mg (na faixa de 165-175 $\left.\mathrm{mg} \mathrm{kg}^{-1}\right)$, e como microelementos o Fe, $\mathrm{Al} \mathrm{e} \mathrm{S} \mathrm{(11,3,}$ 15,9 e $27,8 \mathrm{mg} \mathrm{kg}^{-1}$ ), respectivamente.

Quanto aos macronutrientes analisados, destaca-se o potássio, elemento que apresenta grande mobilidade nas plantas. Devido a sua pouca afinidade em formar quelados orgânicos, explica-se a sua grande quantidade nos tecidos vegetais [26].

Os altos teores encontrados para ferro e alumínio, em comparação aos do cambuci, devem-se, provavelmente, aos constituintes do solo, que nesse caso é típico de cerrado, rico nesses elementos químicos.

TABELA 1 - Composição centesimal dos frutos de gabiroba (C. adamantium), cambuci (C. phaea), jabuticaba (Myrciaria trunciflora), kiwi (Actinidia chinensis), pitanga (Eugenia uniflora), goiaba (Psidium guajava), acerola (Malpighia punicifolia L.), expressas em g $100 \mathrm{~g}^{-1}$.

\begin{tabular}{|c|c|c|c|c|c|c|c|}
\hline Composição & Gabiroba ( $\mathbf{X} \pm \sigma$ ) & Cambuci $^{3}$ & Jabuticaba $^{1,2}$ & Kiwi ${ }^{1,2}$ & Pitanga $^{1,2}$ & Goiaba verm. ${ }^{1,2}$ & Acerola $^{1,5}$ \\
\hline Umidade a $105^{\circ} \mathrm{C}$ & $75,9 \pm 0,1$ & 88,8 & 87,85 & 83,06 & 90,47 & 85,81 & 90,55 \\
\hline Resíduo mineral fixo a $550^{\circ} \mathrm{C}$ & $0,45 \pm 0,01$ & 0,23 & 2,08 & 0,52 & 0,28 & 3,27 & 0,39 \\
\hline Lipídios & $1,5 \pm 0,0$ & 1,5 & 0,89 & 1,37 & 0,23 & 0,64 & $0,30-0,49$ \\
\hline Carboidratos totais* & $11,6 \pm 0,3$ & 5,00 & 8,96 & 13,89 & 8,26 & 9,52 & $7,60-7,79$ \\
\hline Protídeos & $1,6 \pm 0,1$ & 0,44 & 0,22 & 1,16 & 0,76 & 0,76 & $0,40-0,78$ \\
\hline Fibra alimentar & $9,0 \pm 0,0$ & 4,0 & 2,06 & 2,30 & 2,10 & 4,95 & $1,25-2,95$ \\
\hline Ácido ascórbico (Vit. C), mg & $234 \pm 2$ & 33 & 12,8 & 985 & 14,0 & $40,0-80,14$ & 1677,50 \\
\hline Riboflavina (Vit. B2), mg & $0,17 \pm 0,01$ & n.d & - & - & - & - & 0,06 \\
\hline Acidez em ácido cítrico & $1,2 \pm 0,1$ & 3,0 & - & - & - & - & - \\
\hline $\begin{array}{l}\text { pH em solução aquosa, } \\
\left(1: 10 \mathrm{~mL}^{-1}\right), \mathrm{T}=22,2^{\circ} \mathrm{C}\end{array}$ & $4,3 \pm 0,1$ & 2,91 & - & - & - & - & - \\
\hline Valor calórico (kcal $100 \mathrm{~g}^{-1}$ ) & $66,3 \pm 0,6$ & 35,5 & 36,0 & 63,0 & 30,0 & 27,0 & $27,0-31,6$ \\
\hline Óleo essencial, \% (v/p). & 0,06 & $\sim 0,02$ & - & - & - & - & - \\
\hline
\end{tabular}


TABELA 2 - Composição percentual do óleo volátil dos frutos de gabiroba (C. adamantium).

\begin{tabular}{|c|c|c|}
\hline Composto & IK HP-5* & $\%$ \\
\hline 4-hidróxi-4-metil-2-pentanona & 721 & 1,57 \\
\hline tricicleno & 921 & 0,70 \\
\hline$\alpha$-pineno & 930 & 10,61 \\
\hline$\beta$-pineno & 975 & 2,51 \\
\hline$\beta$-mirceno & 984 & 3,53 \\
\hline$\alpha$-felandreno & 998 & 0,35 \\
\hline$p$-cimeno & 1018 & 2,02 \\
\hline limoneno & 1025 & 10,09 \\
\hline (Z)-B-ocimeno & 1035 & 9,22 \\
\hline$\gamma$-terpineno & 1050 & 0,65 \\
\hline Terpinoleno & 1083 & 2,17 \\
\hline Linalool & 1089 & 3,1 \\
\hline terpin-4-ol & 1171 & 1,02 \\
\hline$\alpha$-terpineol & 1175 & 1,94 \\
\hline$\alpha$-copaeno & 1369 & 0,81 \\
\hline$\beta$-elemeno & 1385 & 0,55 \\
\hline$\alpha$-gurjuneno & 1403 & 0,47 \\
\hline$\beta$-cariofileno & 1419 & 7,04 \\
\hline$\beta$-gurjuneno & 1425 & 2,59 \\
\hline$\alpha$-humuleno & 1446 & 2,27 \\
\hline aloaromadendreno & 1455 & 1,03 \\
\hline$\alpha$-amorfeno & 1478 & 0,88 \\
\hline Germacreno-D & 1481 & 1,24 \\
\hline$\beta$-selineno & 1487 & 0,38 \\
\hline Valenceno & 1489 & 0,42 \\
\hline Ledeno & 1495 & 5,16 \\
\hline$\alpha$-muuroleno & 1506 & 0,72 \\
\hline$\gamma$-cadineno & 1511 & 1,38 \\
\hline (Z)-calamaleno & 1521 & 0,45 \\
\hline$\delta$-cadineno & 1522 & 3,15 \\
\hline cadina-1,4-dieno & 1526 & 0,32 \\
\hline$\alpha$-cadineno & 1532 & 0,31 \\
\hline Germacreno B & 1545 & 0,62 \\
\hline$\beta$-maaialeno & 1550 & 0,49 \\
\hline Não identificado (PM = 206) & 1560 & 2,74 \\
\hline Óxido de cariofileno & 1575 & 0,9 \\
\hline Globulol & 1583 & 3,18 \\
\hline Viridiflorol & 1587 & 1,52 \\
\hline Não Identificado (PM = 204) & 1592 & 1,04 \\
\hline$\delta$-cadinol & 1623 & 2,07 \\
\hline$\alpha$-cadinol & 1623 & 0,44 \\
\hline$\tau$-cadinol & 1633 & 1,38 \\
\hline Acetato de farnesila & 1778 & 0,27 \\
\hline Total Identificado & & 93,3 \\
\hline
\end{tabular}

Como contaminantes químicos foram encontrados os elementos $\mathrm{Pb}\left(0,6 \mathrm{mg} \mathrm{kg}^{-1}\right)$ e As $\left(0,7 \mathrm{mg} \mathrm{kg}^{-1}\right)$. Contudo, apenas em sucos de frutas são indicados pela ANVISA [4] e pelo CODEX ALIMENTARIUS [9], os valores máximos de $0,3 \mathrm{mg} \mathrm{kg}^{-1}$ para o $\mathrm{Pb}$ e de $0,2 \mathrm{mg} \mathrm{kg}^{-1}$ para o As, não havendo, portanto, valores referenciados para frutos "in natura".

A presença de contaminantes em amostras "in natura" pode advir do solo e do uso de fertilizantes e/ou agroquímicos em culturas como cana de açúcar e soja, nas regiões próximas a essa unidade de conservação. Em sucos pré-fabricados, deve-se também considerar todo o processamento, envase e armazenagem.

Considerando a contribuição de cada fruto de gabiroba (2,3 g), verificou-se que os frutos dessa região, não apresentaram toxicidade quanto aos teores de metais pesados (Tabela 3), podendo compor a dieta alimentar de vertebrados e serem aproveitados na indústria de alimentos e bebidas.

\section{4 - CONCLUSÃo}

Os frutos de C. adamantium são suculentos, ácidos e levemente adocicados. Apresentam potencial para serem utilizados "in natura", na indústria de alimentos e como flavorizantes na indústria de bebidas, devido aos seus atributos de qualidade como: elevada acidez, ácido ascórbico (vitamina $\mathrm{C}$ ), minerais, fibras alimentares e hidrocarbonetos monoterpênicos ( $\alpha$-pineno, limoneno e $\beta-(z)$ ocimeno), presentes em maior quantidade no óleo volátil dos frutos, e que lhes conferem o aroma cítrico.

\section{5 - REFERÊNCIAS BIBLIOGRÁFICAS}

[1] ADAMS, R. P. Identification of essential oils by ion trap masses spectrometry. New York: Academic Press, 1995. 469 p.

[2] BIBLIOTECA VIRTUAL DO ESTUDANTE BRASILEIRO. Gabiroba. Disponível em: < http:/www.bibvirt.futura. usp.br/especiais/frutasnobrasil/gabiroba.html>. Acesso em: 16 abr. 2004.

[3] BRASIL. Ministério da Saúde. Princípios gerais para o estabelecimento de níveis máximos de ingestão diária em alimentos. Portaria n. 33, de 13 de janeiro de 1998. Diário Oficial da União, Brasília, DF, 16 jan. 1998a. Seção I-E, p. 5.

[4] _____. Princípios gerais para o estabelecimento de níveis máximos de contaminantes químicos em alimento. Portaria n. 685, de 27 de agosto de 1995. Diário Oficial da União, Brasília, DF, 24 set.1998b. Seção 1, n. 183E, p. 3.

[5] ____._. Regulamento técnico geral para fixação dos padrốes de identidade e qualidade para polpa de frutas. Instrução Normativa n.1, de 7 de janeiro de 2000. Diário Oficial da União, Brasília, DF, 10 jan. 2000. Seção 1, p. 54-58.

[6] CABO, J. et al. The spasmolytic activity of various aromatic plants from the province of Granada. I - The activity of major components of their essential oils. Plantes médicinales et phytotherapie, Angers, t. 20, n. 3, p. 213-218, juil. 1986.

[7] CARRARA, M. R. dos. Espécies de Campomanesia Ruiz \& Pavan (Myrtinae, Myrtaceae) ocorrentes no Estado do Rio de Janeiro. Rio de Janeiro, 1997. 222 f. Dissertação (Mestrado em Ciência Biológicas) - Instituto de Biologia, Universidade Federal do Rio de Janeiro, Rio de Janeiro, 1997.

[8] CENTRO DE PESQUISAS ECO-NATURAIS - CEPEN. Cambuci, Campomanesia phaea. Disponível em: $<$ http://www.cepen.com.br/arv_nat_Myrtaceae.htm $>$. Acesso em: 7 out. 2003. 
TABELA 3 - Teores dos elementos inorgânicos nos frutos de gabiroba (C. adamantium), cambuci (C. phaea), jabuticaba (Myrciaria trunciflora), kiwi (Actinidia chinensis), pitanga (Eugenia uniflora), goiaba (Psidium guajava), expressos em $\mathrm{mg} \mathrm{kg}^{-1}$, média de três determinações e respectivos desvio padrão $(\delta)$.

\begin{tabular}{|c|c|c|c|c|c|c|c|}
\hline & Gabiroba (média $\pm \delta$ ) & (\% por fruto) & Cambuci $^{1}$ & Jabuticaba $^{2}$ & $\mathrm{Kiwi}^{2}$ & Pitanga $^{2}$ & Goiaba $^{2}$ \\
\hline \multicolumn{8}{|c|}{ Macroelementos } \\
\hline $\mathrm{Na}$ & $30,7 \pm 0,7$ & 0,007 & 172 & n. d. & 50 & 30 & 30 \\
\hline $\mathrm{K}$ & $1304 \pm 19$ & 0,30 & 623 & 3030 & 3320 & 1030 & 2840 \\
\hline $\mathrm{Ca}$ & $165 \pm 1$ & 0,04 & 61,3 & 130 & 260 & 90 & 200 \\
\hline $\mathrm{Mg}$ & $175 \pm 2$ & 0,04 & 42,1 & n. d. & 300 & 120 & 1,0 \\
\hline $\mathrm{P}$ & $170 \pm 3$ & 0,04 & 124 & 140 & 400 & 110 & 250 \\
\hline \multicolumn{8}{|c|}{ Microelementos } \\
\hline $\mathrm{Ba}$ & $1,1 \pm 0,1$ & 0,0003 & n. d. & n. d. & n. d. & n. d. & n. d. \\
\hline$S$ & $27,8 \pm 0.9$ & 0,006 & n. d. & n. d. & n. d. & n. d. & n. d. \\
\hline $\mathrm{Fe}$ & $11,3 \pm 0,5$ & 0,003 & 3,60 & 19 & 4,1 & 2,0 & 3,1 \\
\hline $\mathrm{Al}$ & $15,9 \pm 0,1$ & 0,004 & 9,6 & n. d. & n. d. & n. d. & n. d. \\
\hline $\mathrm{Zn}$ & $4,9 \pm 0,1$ & 0,001 & 3,46 & n. d. & 1,0 & tr. & 2,3 \\
\hline $\mathrm{Se}$ & $8,8 \pm 0,2$ & 0,002 & n. d. & n. d. & n. d. & tr. & tr. \\
\hline $\mathrm{Ni}$ & $0,2 \pm 0,0$ & 0,00005 & 0,17 & n. d. & n. d. & n. d. & n. d. \\
\hline $\mathrm{Cu}$ & $1,9 \pm 0,1$ & 0,0004 & 1,46 & n. d & 0,5 & 2,0 & 1,0 \\
\hline $\mathrm{Pb}$ & $0,6 \pm 0,2$ & 0,0001 & 1,63 & n. d. & n. d. & n. d. & n. d. \\
\hline As & $0,7 \pm 0,2$ & 0,0002 & n. d. & n. d & n. d. & n. d. & n. d. \\
\hline $\mathrm{Mn}$ & $2,1 \pm 0,2$ & 0,0005 & 0,27 & n. $d$ & 1,0 & tr. & 1,4 \\
\hline
\end{tabular}

Vallilo [30]; ${ }^{2}$ Philippi [28]; n. d. = - não detectado; tr.- traço.

[9] CODEX ALIMENTARIUS COMISSION. General standard for contaminants and toxins in foods. Rome: FAO/WHO, 1996. p. 4-16. (CODEX ALIMENTARIUS, SCHEDULE I).

[10] CONTRERAS-GUZMÁN, E. S.; STRONG III, F. C.; GUERNELLI, O. Determinação de ácido ascórbico (vitamina C) por redução de ions cúpricos. Química Nova, São Paulo, v. 7, n. 2, p. 60-64, abr. 1984.

[11] CROWELL, P. L.; SIAR AYOUBI, A.; BURKE, Y. D. Antitumorigenic effects of limonene and perillyl alcohol agains pancreatic and breast cancer. Adv Exp Med Biol., New York, v. 401, p. 131-6, 1996.

[12] DE ANGELIS, R. C. Fisiologia da nutrição: fundamentos para nutrição e desnutrição. São Paulo: EDART, 1977. v. 1 , p. $43-53$

[13] DURIGAN, G. et al. Regeneração natural da vegetação de cerrado sob floresta de Eucalyptus citriodora. Rev. Inst. Flor., São Paulo, v. 9, n. 1, p. 71-85, jul. 1997.

[14] DURIGAN, G. et al. Plantas do cerrado paulista: imagens de uma paisagem ameaçada. São Paulo: Páginas \& Letras, 2004. $475 \mathrm{p}$.

[15] FRANCO, G. Tabela de composição química de alimentos. 9. ed. Rio de Janeiro: Atheneu, 1992. 305 p.

[16] FRANCO, M. R. B. Aroma e sabor de alimentos: temas atuais. São Paulo: Livraria Varela, 2003. 246 p.

[17] GARRIDO, M. A. de O. et al. Estação Experimental e Ecológica de Assis. São Paulo: Páginas \& Letras, 1997. $15 \mathrm{p}$.

[18] _. Florestal Estadual de Assis e Estação Ecológica de Assis. Assis: Floresta Estadual de Assis e Estação Ecológica de Assis, Instituto Florestal, SP. 2004. 22 p.

[19] GHELARDINI, C. et al. Local anaesthetic activity of $\beta$-caryophyllene. Il Fármaco, Milan, v. 56, n. 3, p. 387389, May-July 2001.
[20] INSTITUTO ADOLFO LUTZ. Normas analíticas do Instituto Adolfo Lutz. 3. ed. São Paulo: IMESP, 1985. v. $1,533 \mathrm{p}$.

[21] KAWAMORI, T. et al. Inhibitory effects of d-limonene on the development of colonic aberrant crypt foci induced by azoxymethane in F344 rats. Carcinogenesis, London, v. 17, n. 2, p. 369-72, Feb. 1996.

[22] LAJOLO, M. F. Tabela brasileira de composição de alimentos. Disponível em: <www.fcf.usp.tabela $>$. Acesso em: 3 dez. 2001.

[23] LARCHE, W. Ecofisiologia vegetal. São Carlos: Rima, 2000. $531 \mathrm{p}$.

[24] LEE, S. C.; PROSKY, L.; DEVRIES, J. W. Determination of total, soluble and insoluble dietary fiber in foods. Enzimatic-gravimetric method, Mês-TRI Buffer: collaborative study. J. Assoc. Off. Anal. Chem. Int., Gaithersburg, v. 75, n. 3, p. 395-416, May-June 1992.

[25] LORENZI, H. Árvores brasileiras: manual de identificação e cultivo de plantas arbóreas nativas do Brasil. Nova Odessa: Plantarum, 1992. 252 p.

[26] MACARI, P. A T. et al. Composição entre os metais presentes em Croton floribundus Spreng. e Baccharis dracunculifolia DC. Rev. Bras. Farmacogn., São Paulo, v. 12, p. 76-77, 2002 (supl.).

[27] MARTIN, S. et al. Anti-inflammatory activity of the essential oil of Bupleurum fruticescens. Planta Med., Studgard, v. 59, p. 533-36, Dec. 1993.

[28] PHILIPPI, S. T. Tabela de composição de alimentos: suporte para decisão nutricional. Brasília, DF: Anvisa, Finatec/Nut-UnB, 2001. 133 p.

[29] VALLILO, M. I. et al. Composição química do fruto de Eugenia klotzschiana Berg. (MYRTACEAE). Rev. Inst. Flor., São Paulo, v. 15, n. 1, p. 33-44, jun. 2003.

[30] VALLILO, M. I. et al. Características físicas e químicas dos frutos do cambucizeiro (Campomanesia phaea). 
Rev. Bras. Frutic., Jaboticabal, v. 27, n. 2, p. 241-244, ago. 2005.

[31] WASICKY, R. Uma modificação do aparelho de Clevenger para extração de óleo essencial. Rev. Fac. Farm. e Bioq., São Paulo, v. 1, n. 1, p. 77-81, jan.-jun.1963.

\section{6 - AGRADECIMENTOS}

Aos funcionários José Benedito Lourenço e João Barbosa da Silva, trabalhadores braçais da Unidade de Conservação de Assis, pelo auxílio na coleta do material botânico.

Ao CNPq / FAPESP pelo suporte financeiro. 